ORIGINAL RESEARCH

\title{
Identification of risk factors for exertional heat-related illnesses in long-distance cyclists: experience from the California AIDS Ride
}

\author{
MICHELLE A. KRUEGER-KALINSKI, MD; DAVID L. SCHRIGER, MD, MPH; LAURENCE FRIEDMAN, \\ MD; SCOTT R. VOTEY, MD
}

From the University of California, Los Angeles (UCLA) Emergency Medicine Center, UCLA School of Medicine, Los Angeles, CA.

\begin{abstract}
Objectives. - To identify risk factors for exertional heat-related illnesses and to determine if patients who are afflicted with the human immunodeficiency virus or have acquired immunodeficiency syndrome have an increased risk of exertional heat-related illness.

Design.-Descriptive, retrospective, matched case-control study.

Setting.-Mobile community of 2650 participants in the California AIDS Ride 3.

Participants.-Participants included 117 patients presenting for medical care with heat-related illnesses and 234 age-, gender-, and registration site-matched control subjects who did not develop a heat-related illness.

Methods.- Retrospective, matched case-control study utilizing univariate and multivariate conditional logistic regression to determine if human immunodeficiency virus seropositivity, the number of chronic medical illnesses, or the number of current medications known to affect heat dissipation increased the risk of exertional heat-related illnesses.

Results. - The multivariate model revealed that patients with a greater number of chronic medical illnesses were at a significantly increased risk of a heat-related illness (odds ratio $=1.6,95 \% \mathrm{CI}=$ 1.2-2.1). The number of current medications (odds ratio $=1.1,95 \% \mathrm{CI}=0.8-1.5$ ) and human immunodeficiency virus seropositivity (odds ratio $=0.7,95 \% \mathrm{CI}=0.2-3.1$ ) were not significant predictors of risk for exertional heat-related illness.

Conclusions. - Those riders suffering from a greater number of chronic medical illnesses were at a greater risk for developing an exertional heat-related illness. Human immunodeficiency virus seropositivity alone was not associated with increased risk of exertional heat-related illness.
\end{abstract}

Key words: heat illness, heat injury, cycling, human immunodeficiency virus, acquired immunodeficiency syndrome, AIDS, HIV

\section{Introduction}

Previous studies have determined risk factors for nonexertional heat-related illnesses (primarily classic nonexertional heatstroke) and exertional heat-related illness in healthy subjects. ${ }^{1-12}$ Many studies and textbooks address the differences between classic and exertional heatstroke. It is frequently taught that classic heatstroke is more frequent in the elderly, chronically ill, and sedentary population on multiple medications, whereas exertional heatstroke is more common in young healthy males exposed

Corresponding author: Michelle Krueger-Kalinski, MD, Community Hospital of the Monterey Peninsula, PO Box HH, Monterey, CA 93940 (e-mail michkruege@aol.com). to exertional stress in hot conditions. ${ }^{1-12}$ Since the reports of exertional heat illnesses most commonly deal with athletes or the active-duty military population, it is not surprising that these studies might be limited by having a relatively healthy population base. ${ }^{6-12}$

The California AIDS Ride offered a unique environment in which to study heat-related illnesses. The population includes a large number of chronically ill patients on multiple medications. Participants may or may not be competitive athletes, and training regimens are quite variable. We hypothesized that when exposed to extreme temperatures and vigorous exercise, those individuals with a greater number of chronic medical conditions, those requiring multiple medications, and patients with 
acquired immunodeficiency syndrome (AIDS) would have a greater risk of developing an exertional heat-related illness, similar to what has been experienced in classic heatstroke. ${ }^{2}$

The purpose of this study was to determine if risk factors for exertional heat-related illnesses could be identified in a group of healthy and chronically ill individuals participating in a multiple-day-long distance cycling event. We hypothesized that the risk of heatrelated illnesses would be correlated with the number of chronic medical conditions, the number of medications that diminish heat dissipation, and the human immunodeficiency virus (HIV) status of the rider, and we conducted a retrospective, matched case-control study to examine these hypotheses.

\section{Materials and methods}

\section{THE EVENT}

California AIDS Ride 3 was a 547-mile bicycle ride from San Francisco, CA, to Los Angeles, CA, which occurred between June 1 and June 8, 1996. There were 2650 participants in the 7-day event, including $2250 \mathrm{cy}$ clists and 400 support staff (noncyclists). Riders and support staff spent nights in a tent camp that was moved each day by the support staff while the riders were in transit.

Individuals with multiple medical problems and chronic illnesses (including patients needing total parenteral nutrition and daily intravenous medications) were allowed to participate in the ride. Prior to the event, all participants were required to provide a written, self-reported medical history, including age, gender, surgical history, hospitalizations, chronic medical illnesses, current review of systems, current prescription and overthe-counter medications, a description of any disability lasting more than 3 days, HIV status (optional), AIDS status (optional), family history, vaccination history, alcohol use, and, for women, last menstrual period.

Medical care was continuously available to riders and support staff throughout the 7-day event. During cycling hours, care was provided by 2 roaming advanced life support (ALS) ambulances staffed by a physician, a paramedic, and an emergency medical technician; an ALS-equipped medical vehicle with a 4-bed capacity; and 4 stationary first aid stations staffed by nurses. In camp, medical care was provided at an ALS-capable facility staffed with physicians, nurses, paramedics, and emergency medical technicians. The mobile 4-bed vehicle and the base camp were used to provide comprehensive treatment of heat-related illnesses, including intravenous hydration; evaporative and conductive cool- ing; and oral, rectal, intramuscular, and intravenous antiemetics. Patients who did not respond to these measures were transferred to local emergency departments. A medical record was created for all patients seen by an event physician.

\section{IDENTIFICATION OF CASES AND CONTROLS}

Medical records of all encounters with event physicians were examined by one of the authors, and all episodes of heat-related illness were identified. Patients with visits for heat-related illnesses were deemed "cases." Patients could have more than 1 visit for a heat-related illness. Heat-related illnesses included heat cramps, heat syncope, heat edema, heat exhaustion, and heatstroke (defined as an unexplained altered mental status in association with significant heat exposure). ${ }^{2}$ Two matched controls were identified for each case by randomizing the order of all non-case-related, self-reported medical histories and selecting the first 2 subjects who registered at the same location (Los Angeles or San Francisco), held the same role (cyclist vs noncyclist), were the same gender, and were within 1 year of age of the case. One case involved a woman from Los Angeles who was observing the final ceremonies (neither a cyclist nor staff) and sought care for a heat-related illness. Controls for this patient were taken from the staff category.

\section{DATA ABSTRACTION}

For each heat-related physician encounter, we abstracted the following information from the medical record: vital signs, whether intravenous fluids were used, whether transfer to an emergency department was required, whether the patient was hospitalized, and the field physician's diagnosis. For cases with more than 1 encounter we made a separate abstraction of each encounter.

For all cases and controls we directly abstracted their age, gender, HIV status, registration area (as most training was carried out near the site of registration), and role in the event (cyclist vs noncyclist). We then counted the number of chronic medical illnesses (diabetes, hypertension, asthma, heart disease, cancer, AIDS, seizure disorders, etc) and the number of current medications that could diminish the body's ability to handle heat stress (anticholinergics, sympathomimetics, thyroid hormone, diuretics, cardiac medications, cyclic antidepressants, phenothiazines, antihistamines, etc) for each case and control.

Due to potential inconsistencies in diagnostic criteria for heat-related illnesses among the event physicians, we developed a heat illness severity score based on criteria that could be objectively obtained from a retrospective 
Descriptive data on patients with exertional heat-related illnesses*

\begin{tabular}{lrrr}
\hline & Cases & Controls & Total population \\
\hline Total no. & 117 & 234 & 2650 \\
Mean age, y (SD) & $34 \quad(8)$ & $35 \quad(9)$ & $35(9)$ \\
Female, \% & 64 & 64 & 42 \\
Cyclist, \% & 91 & 92 & 85 \\
Registered in Los Angeles, \% & 55 & 55 & 33 \\
HIV positive, \% (total respondents) & $8 \quad(101)$ & $0.4(1.0)$ & $\ldots$ \\
Average no. of medications (SD) & $0.8(1.4)$ & $0.8(0.9)$ & $\ldots$ \\
Average no. of illnesses (SD) & $1.3(1.3)$ & $\ldots$ & $\ldots$ \\
Requiring IV fluids, no. (\%) & $38 \quad(33 \%)$ & $\ldots$ & $\ldots$ \\
Emergency visits, no. (\%) & $22 \quad(19 \%)$ & $\ldots$ & $\ldots$ \\
Hospitalized, no. (\%) & $6 \quad(4 \%)$ & & $\ldots$ \\
\hline
\end{tabular}

* HIV indicates human immunodeficiency virus; IV, intravenous.

chart review. The heat-related illness was scored as severe if hospitalization was required, moderate if intravenous fluid resuscitation or transfer to an emergency department was required, and mild if it was managed solely with oral hydration and cooling measures.

\section{DATA ANALYSIS}

Standard descriptive statistics were calculated on both a by-person and by-medical encounter basis. A univariate conditional fixed-effects logistic regression (STATA 5.0, STATA Corp., College Station, TX) was used to examine the explanatory power of the number of chronic illnesses, number of current medications, and the HIV status in differentiating cases from controls. Multivariate conditional fixed-effects logistic regression analysis of the same variables was used to assess the explanatory power of these variables.

\section{Results}

Maximum daily ambient temperature varied from $26.7^{\circ} \mathrm{C}$ to $43.3^{\circ} \mathrm{C}$. The distribution of visits for heat-related illness closely followed the distribution of daily ambient temperatures. During the week-long event 118 individuals presented for care of 142 episodes of heat-related illness that warranted examination by a physician. One patient (who had 1 visit) was omitted from the analysis because the patient's age was not documented, leaving 117 patients with 141 heat-related illnesses available for analysis. The average age of cases (34.4 \pm 8 years) was similar to that for all participants (34.7 \pm 9 years). Women accounted for $64 \%$ of the cases, and $42 \%$ of all participants $(\Delta=22 \%, 95 \%$ CI $13 \%-31 \%)$. Nine $(2.3 \%)$ of the 400 support staff and $107(4.8 \%)$ of the 2250 cyclists became cases $(\Delta=2.5 \%, 95 \%$ CI $0.8 \%-4.2 \%)$.
The average case had 1.2 visits for heat-related illnesses (Table).

Sixty-eight percent (97/141) of visits were classified as mild, and $95 \%$ of these visits were for heat exhaustion. Twenty-seven percent of visits were classified as moderate (16 requiring intravenous hydration and 22 requiring intravenous hydration and transfer to an emergency department), and $97 \%$ were for heat exhaustion. The remaining visit was for heat syncope. Four percent (6 of the 141 visits) of the patients required hospitalization. Three patients were hospitalized for heat exhaustion, and 3 for heatstroke.

Univariate conditional logistic regression analysis for matched case-control groups was performed on the following variables: HIV status (positive or negative), number of medications diminishing heat dissipation, and number of chronic medical illnesses. Human immunodeficiency virus status was available for 101 of the 117 cases and for 218 of the 234 controls. The number of chronic medical illnesses (odds ratio $=1.6,95 \% \mathrm{CI}=$ $1.3-2.1, P<.001)$ and the number of current medications $($ odds ratio $=1.5,95 \% \mathrm{CI}=1.1-1.9, P=.002$ ) both significantly predicted an increased risk of heat illness. Human immunodeficiency virus status did not predict heat illness (odds ratio $=2.3,95 \% \mathrm{CI}=0.7-7.4$, $P=.15)$.

When these variables were used in a multivariate conditional logistic regression analysis, only the number of chronic medical illnesses was significantly associated with a predisposition to heat-related illnesses (odds ratio $=1.6,95 \% \mathrm{CI}=1.2-2.1, P=.002)$. The number of medications (odds ratio $=1.1,95 \% \mathrm{CI}=0.8-1.5, P=$ $.63)$ was no longer predictive. Although there was a tendency toward a relationship, the HIV status (odds ratio $=0.7,95 \% \mathrm{CI}=0.2-3.1, P=.65$ ) was not a significant 
negative predictor of a heat-related illness. Results varied little when the multivariate model was rerun with the number of illnesses recorded as 1 or less (0) or 2 or more (1).

To determine if missing HIV data could affect the results, we reanalyzed the data assuming that cases and controls with missing HIV status were either all positive or all negative. In both cases the results were essentially unchanged.

\section{Discussion}

We investigated the risk factors for exertional heat-related illness in a large population subject to repetitive, strenuous physical exertion at high ambient air temperatures. Our study population differed from those in previous studies of exertional heat illness, as many of the participants had a large number of chronic medical illnesses, were on multiple medications, or were HIV positive. We hypothesized that HIV seropositivity, the number of chronic medical illnesses, and the number of medications would increase the risk of a heat-related illness. We found, using univariate conditional logistic regression analysis, that both the number of chronic medical illnesses and the number of current medications significantly predicted an increased risk of heat-related illnesses. Human immunodeficiency virus seropositivity did not increase the risk of heat illness. However, using multivariate conditional logistic regression analysis only, the number of chronic medical illnesses was significantly associated with heat-related illnesses, verifying a portion of our initial hypothesis.

Heat-related illnesses are partially immune-related processes. Previous immunologic studies on exertional heatstroke showed large increases in interferon, tumor necrosis factor, and interleukins 1,6 , and 2 r (IL-1, IL6 , and IL-2r). ${ }^{2,13}$ Exercise has been shown to increase overall leukocyte counts, increase cortisol, and decrease the concentration of CD3, CD16, and CD19 cells. ${ }^{2,14}$ Intense training and competition has resulted in reports of some athletes becoming relatively immune suppressed and more susceptible to infection. ${ }^{2,14}$ Previous studies evaluating the effects of exercise on patients with AIDS have yielded conflicting results. Ullum et al ${ }^{15}$ found that HIV-positive individuals had an impaired ability to mobilize neutrophils, natural killer cells, and lymphokineactivated killer cells. The absolute CD4 count was decreased, but the percentage of $\mathrm{CD} 4$ cells was unchanged. A review of the literature by LaPerriere et $\mathrm{al}^{16}$ found no decline in CD4 cell counts and a trend toward an increase in CD4 counts. A third study by Lawless et al ${ }^{17}$ noted that maximal exercise (like that experienced in the AIDS Ride) weakens nonspecific cell immunity, de- creases neutrophil activity, decreases CD4+/CD8+ ratios, and decreases B-cell lymphocyte function. Since HIV-positive patients frequently are immunosuppressed, it was hypothesized that they might have greater difficulty with maximal exercise, such as that experienced in the California AIDS Ride. We hypothesized that they would have an increased risk of heat-related illnesses. On the univariate analysis, the HIV variable was a nonsignificant predictor of an increased risk of heat illness. On the multivariate analysis, the "number of illnesses" variables presumably accounted for those patients who had AIDS, and the coefficient of the HIV variable reflects the effect of being HIV-positive without AIDS. In this multivariate analysis the HIV variable was a nonsignificant negative predictor of heat illness. Patients with HIV might have experienced fewer heat-related illnesses than we anticipated because of their training efforts. For example, a group of identified HIV-positive participants ("The Positive Pedallers") were known to be thoroughly prepared, moderate in their exertion, and attentive to rehydration.

The findings in this study have implications for medical care providers at future athletic events that include participants individuals with chronic medical illnesses and exposure to heat stress. Medical providers must use caution accepting participants with multiple chronic medical illnesses. If pre-event participant screening identifies a large number of participants at increased risk for heat-related illness, one or more of the following alternatives should be considered: 1) modifying the event to decrease exertion levels or heat stress, 2) increasing the number of medical support staff, 3) educating participants at high risk for heat-related illness on the prevention and warning signs of heat-related illnesses, 4) developing policies and procedures to closely monitor participants with mandatory cessation of active participation if early evidence of a heat-related illness is identified, and 5) development of policies and procedures for mandatory cessation of active participation in the event if extreme heat conditions occur.

This study has several limitations. First, although the structure of the event resulted in all patients with moderate or severe heat illness being seen by an event physician, individuals with mild heat illness who appear well may not have presented or been brought to medical attention, potentially resulting in an underestimate of the incidence of heat illness. Second, pertinent data were not always adequately obtained or were incompletely recorded on the patient encounter sheets. Core temperatures were rarely obtained, and cooling measures were often initiated prior to obtaining any temperature measurement. Although we chose specific identifiable management interventions to define our categories of mild, 
moderate, and severe heat illness, the lack of core temperatures and the early initiation of therapy may have resulted in a failure of event physicians to recognize the severity of the heat illness. This might have resulted in event physicians limiting more aggressive treatment, which might have resulted in an underestimate of the incidence of more serious heat illnesses. Third, the event physicians may have differed in their application of the severity-defining therapeutic interventions, resulting in either overestimates or underestimates of the severity of heat illness. Finally, utilizing a self-reported medical history risks a failure of the participants to report all medical conditions and medications, potentially resulting in a lower odds ratio and an underestimate of the positive predictive power of these factors.

In conclusion, we found that most participants, including those with multiple chronic medical illnesses, were able to successfully participate in an athletic event requiring repetitive strenuous exertion and severe heat stress. A small number of participants (4.5\%), however, developed a clinically important heat-related illness. Since these events will continue to occur and will require medical support, appropriate logistical and medical planning will be necessary. With appropriate medical support, most participants can safely complete events similar to the California AIDS Ride 3 without developing a significant heat-related illness. Specifically, we found no basis to discourage HIV-positive individuals without AIDS from active participation in similar events. Although this group is not typical of the general HIV-positive patient population, they are probably typical of HIV-positive patients who would enter a race like this. We would recommend that all physicians counseling patients on events such as this clearly define training requirements and potential risks to their patients.

\section{References}

1. Clowes GHA Jr, O'Donnell TF Jr. Heat stroke. $N$ Engl $J$ Med. 1974;291:564.

2. Hubbard RW, Gaffin SL, Squire DL. Heat-related illnesses. In Auerbach PS, ed. Wilderness Medicine: Manage- ment of Wilderness and Environmental Emergencies. 3rd ed. St Louis, MO: Mosby; 1995:167-212.

3. Khogali M. Epidemiology of heat illnesses during the Makkah pilgrimages in Saudi Arabia. Int J Epidemiol. 1983;12:267-273.

4. Kilbourne EM, Choi K, Jones TS, Thacker SB. Risk factors for heatstroke: a case-control study. JAMA. 1982;247: 3332-3336.

5. Semanza JC, Rubin $\mathrm{CH}$, Falter $\mathrm{KH}$, et al. Heat-related deaths during the July 1995 heat wave in Chicago. $N$ Engl J Med. 1996;335:84-90.

6. American College of Sports Medicine. Prevention of thermal injuries during distance running (position statement). Physician Sports Med. 1984;12(7):43-51.

7. Barthel Herman J. Exertion-induced heatstroke in a military setting. Mil Med. 1990;155:116-119.

8. England AC III, Fraser DW, Hightower AW, et al. Preventing severe heat injury in runners: suggestions from the 1979 Peach Tree Road Race experience. Ann Intern Med. 1982;97:196-201.

9. Knochel JP. Environmental heat illness: an eclectic review. Arch Intern Med. 1974;133:841.

10. Murphy RJ. Heat illness in the athlete. Am J Sports Med. 1984;12:258-261.

11. Porter AM. Heat illness and soldiers. Mil Med. 1993;158: 606-609.

12. Sutton JR, Hughson RL. Heatstroke in road races. Lancet. 1979;1:983.

13. Sprenger H, Jacobs C, Nain M, et al. Enhanced release of cytokines, interleukin-2 receptors, and neopterin after long-distance running. Clin Immunol Immunopathol. 1992; 63:188-195.

14. Haq A, al-Hussein K, Lee J, al-Sedairy S. Changes in peripheral blood lymphocyte subsets associated with marathon running. Med Sci Sports Exerc. 1993;25:186-190.

15. Ullum H, Palmo J, Halkjaer-Kristensen J, et al. The effect of acute exercise on lymphocyte subsets, natural killer cells, proliferative responses, and cytokines in HIV-seropositive persons. J Acquir Immune Defic Syndr. 1994;7: $1122-1133$.

16. LaPerriere A, Klimas N, Fletcher MA, et al. Change in CD4+ cell enumeration following aerobic exercise training in HIV-1 disease: possible mechanisms and practical applications. Int J Sports Med. 1997;18(suppl 1):S56-61.

17. Lawless D, Jackson CGR, Greenleaf JE, et al. Exercise and human immunodeficiency virus (HIV-1) infection. Sports Med. 1995;19:235-239. 